JOURNAL OF SYNCHROTRON RADIATION

ISSN 1600-5775

Received 19 February 2016

Accepted 27 April 2016

Edited by J. F. van der Veen

Keywords: free-electron laser; undulator beamline design; simulations.

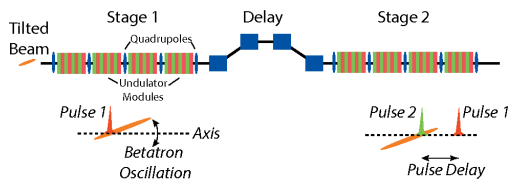

(C) 2016 International Union of Crystallography

\section{Two-color operation of a free-electron laser with a tilted beam}

\author{
Sven Reiche* and Eduard Prat
}

Paul Scherrer Institute, CH-5232 Villigen PSI, Switzerland. *Correspondence e-mail: sven.reiche@psi.ch

With the successful operation of free-electron lasers (FELs) as user facilities there has been a growing demand for experiments with two photon pulses with variable photon energy and time separation. A configuration of an undulator with variable-gap control and a delaying chicane in the middle of the beamline is proposed. An injected electron beam with a transverse tilt will only yield FEL radiation for the parts which are close to the undulator axis. This allows, after realigning and delaying the electron beam, a different part of the bunch to be used to produce a second FEL pulse. This method offers independent control in photon energy and delay. For the parameters of the soft X-ray beamline Athos at the SwissFEL facility the photon energy tuning range is a factor of five with an adjustable delay between the two pulses from -50 to $950 \mathrm{fs}$.

\section{Introduction}

Free-electron lasers (FELs) provide femtosecond light pulses with transverse coherence and freely tunable central photon energy, thus representing essential tools for time-resolved experiments to a broad range of research fields in science such as structural biology, chemistry, surface physics and condensed matter. Thanks to photon pulse lengths of the order of femtoseconds, pump-probe experiments can achieve unprecedented time resolution for any kind of ultrafast dynamics. The common approach is to pump with an external signal to initiate the reaction of the process to be investigated. Most of these pump signals can be generated by an external pump laser system, which can cover a broad range of photon energies from THz (Shalaby \& Hauri, 2015) through IR and visible to VUV (Ravasio et al., 2009). However, some experiments call for pump and probe signals that are both in the X-ray regime, such as stimulated Raman scattering (Schweigert \& Mukamel, 2007) or simultaneously time-resolved measurements of different electron states (Dlott \& Fayer, 1989). Because both pulses are in the X-ray photon energy range, where no efficient external sources exist, they have to be derived from the same undulator beamline.

Concerning the generation of two-color XFEL pulses, one possibility is to use a series of undulator modules alternately tuned to two well separated resonant frequencies (by different undulator parameters $K_{1}$ and $K_{2}$ ) with a quasi-mono-energetic electron beam. This idea has been studied theoretically and proved experimentally (see, for instance, Geloni et al., 2010a,b; Lutman et al., 2013; Marinelli et al., 2013; Hara et al., 2013; Campbell et al., 2014), but has some weaknesses: each color utilizes only half of the undulator line and both colors are generated by the same bunch, so it is more difficult for the two pulses to reach saturation at the same power level.

A way to overcome these limitations involves the generation of an electron beam with different energy bands sent 
through an undulator with a given undulator parameter. The resonant wavelength is then defined by the local beam energy. In this manner each color uses all the undulator modules, thus minimizing the length of the undulator beamline, and both colors can easily saturate. This has been achieved by generating at the source two electron beamlets with adjustable time and energy spacing, at optical wavelengths (Petrillo et al., 2013; Ronsivalle et al., 2014) and recently also in the X-ray regime (Marinelli et al., 2015). Seeding techniques can be applied to reduce the bandwidth of each color (Marinelli et al., 2015). By using a quasi-mono-energetic beam and one or more self-seeding monochromator crystals it is possible to generate two-color pulses with ultra-narrow bandwidth in the hard X-ray regime (Geloni et al., 2011; Lutman et al., 2014). In laserbased seeding schemes, the two beamlets can be generated by the seed laser (Allaria et al., 2013), or the two-color XFEL pulses can be generated by using a powerful laser seed with an energy chirp (De Ninno et al., 2013; Mahieu et al., 2013).

The fundamental challenge lies in the control of the photon energies and the time separation, preferably independent of each other. Most of the proposed methods restrict the generated pulses within the limit of the bunch duration, most of the time even with a strong correlation between time separation and photon pulse energies. In addition, tuning the photon energy by a variable-gap undulator in a two-stage process is much more flexible than with a fixed gap and an energy chirp along the electron bunch. In addition, a large correlated energy spread yields chromatic effects in the electron bunch transport ultimately limiting the tunability to a few tenths of a percent.

We describe an improved method where two parts of the same bunch produce two pulses. The selection of the photon energy is made by changing the undulator gap. To avoid the problem that both parts of the bunch are amplifying radiation in a single stage, the beam is tilted and misaligned so that only one part is lasing. In the delaying chicane the second part of the beam is realigned to generate the second pulse. As a reference the chosen beam and FEL parameters are similar to the soft-X-ray FEL beamline Athos at SwissFEL (Ganter, 2012), but can easily be scaled to different wavelength ranges such as hard X-rays or VUV.

\section{Undulator beamline configuration for two-color operation}

To provide two FEL pulses with a large flexibility in the relative delay with respect to each other and the central wavelength of each pulse, while keeping the other FEL parameters comparable (e.g. pulse energy or pulse length), a two-stage configuration of the undulator is proposed. In between the stages a magnetic chicane can delay the electron bunch. The primary idea is that, due a tilt in the electron beam, only one part of the electron bunch is lasing at a given time. Within the delaying chicane the bunch is re-aligned transversely so that the second stage operates on a fresh part of the bunch. The wavelengths are selected by tuning the undulator parameter to the desired values. With sufficiently large values the tunability is more flexible than by tuning with the central electron beam energy, where the limit is given at roughly $10 \%$ in beam energy and $20 \%$ in FEL photon energy, respectively, before electron energy collimation, spurious dispersion and chromatic effects prevent the FEL from amplifying the signal equally well for both pulses (Widmann et al., 2014). A schematic layout is shown in Fig. 1.

Our example layout follows closely the design of the soft X-ray FEL beamline Athos at the SwissFEL facility, located at the Paul Scherrer Institute, and the simulations presented in this paper are based on those beam and undulator parameters with an electron beam energy of 2.5 to $3.5 \mathrm{GeV}$, a bunch length of $100 \mathrm{fs}$ and a tunablity of the undulator parameter between 1 and 3.5 (see Table 1). The baseline design foresees the operation as a SASE-FEL and the improvement of the spectral flux by means of self-seeding very similar to the configuration at LCLS (Ratner et al., 2015). From the self-seeding option the delaying chicane of the electron bypass is used to control the relative arrival time of the second FEL pulse. The maximum delay is $1 \mathrm{ps}$ for the $3 \mathrm{GeV}$ electron bunch. Each stage of the undulator is about $30 \mathrm{~m}$ long, consisting of an alternating array of short undulator modules of $2 \mathrm{~m}$ length and quadrupole magnets. The latter provide sufficient focusing strengths close to the stable limit of the beam transport. A rather short undulator module length and thus a small average betatron-function facilitates the two-color operation discussed in this paper by a faster betatron-oscillation of the misaligned parts of the bunch and therefore a more efficient suppression of the lasing process (Reiche, 2000). The systematic study of the beam tilt and betatron-function is described in $\S 4$.

The design of the SwissFEL undulator provides a practical variation of the undulator parameter between 1 and 3.5 , where values below 1 are excluded due to the significant increase in the gain length and thus not providing saturation within the given length of the undulator. This corresponds roughly to a tuning range in photon energy of a factor 4.75 for a fixed energy of the electron beam. As an example of the flexibility in selecting the photon energy we choose the reference photon energies of $282 \mathrm{eV}$ and $533 \mathrm{eV}$ for our simulations, corresponding to the $K$-edges of carbon and oxygen, respectively. The photon energy difference fits well within the tuning range

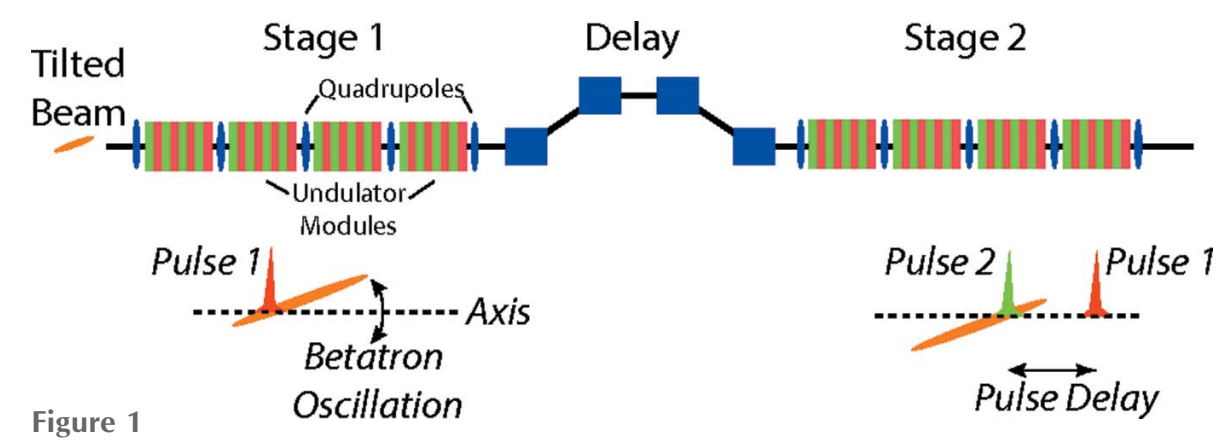


of the Athos undulator but it is too large to achieve it by means of beam energy differences alone, as proposed in other two-color schemes. The difference in energy would be $37 \%$, yielding technical challenges in this case for beam transport and simultaneous matching to the undulator lattice. Also, the ratio of the photon energies is not close to an integer, which could have been provided rather easily by different steps in a high-gain harmonic-generation FEL cascade (Yu \& BenZvi, 1997).

\section{Generation of the beam tilt}

The electron beam parameters are listed in Table 1, following the baseline design of SwissFEL. The required tilt can be introduced by various methods. The most straightforward procedures are the use of a transverse deflecting structure or residual dispersion from compression stages before the undulator beamline. Both methods cause a degradation of the beam quality. In the case of dispersion the size of the beam slices is increased because the intrinsic energy spread shears the beam transversely. For an upper limit of a $2 \mathrm{~mm}$ tilt and a full width of $2 \%$ energy variation a dispersion function value of $\eta=10 \mathrm{~cm}$ is needed. For SwissFEL parameters with an intrinsic energy spread of $250 \mathrm{keV}$ and a beam energy of $3 \mathrm{GeV}$ the shearing is $8.3 \mu \mathrm{m}$. Convolved with the intrinsic beam size of $22.6 \mu \mathrm{m}$ from start-to-end simulations the effective beam size is $24.1 \mu \mathrm{m}$. This relative increase of $6.5 \%$ in beam size can be modeled with an emittance value larger by $13 \%$ in the streaking plane. Even with this slight increase, results from start-to-end simulations yield still smaller emittance values than the design value for SwissFEL of $430 \mathrm{~nm}$ (Ganter, 2012), which was used to estimate the minimum required length of the undulator to guarantee saturation. Alternatively a transverse deflecting RF structure can tilt the beam. The drawback of such a device is the off-axis electric field in the structure, which increases the energy spread. For SwissFEL beam parameters a $2 \mathrm{~mm}$ tilt within the undulator would require an RF field increasing the r.m.s. energy spread

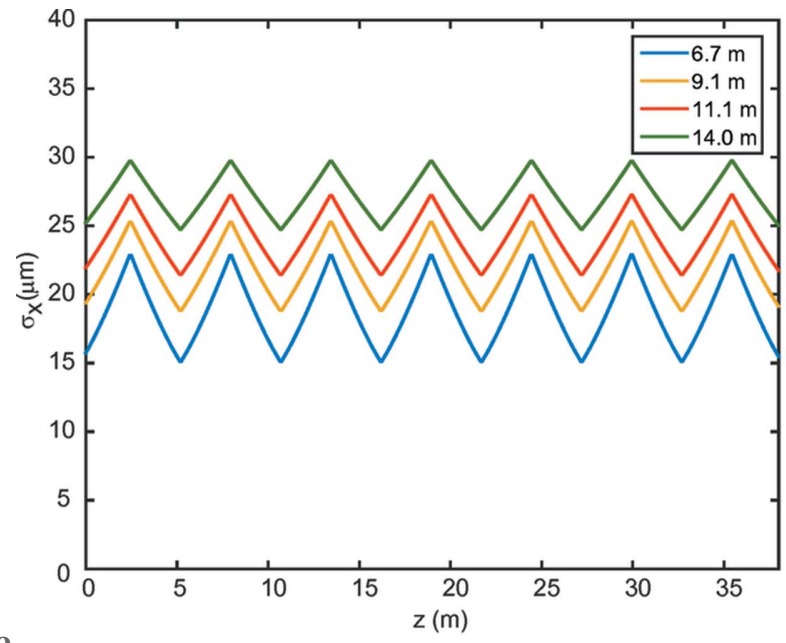

Table 1

Beam parameters used for simulations.

\begin{tabular}{ll}
\hline Parameter & Value \\
\hline Energy & $2.91 \mathrm{GeV}$ \\
Energy spread & $460 \mathrm{keV}$ \\
Emittance & $300 \mathrm{~nm}$ \\
Current & $2000 \mathrm{~A}$ \\
Charge & $200 \mathrm{pC}$ \\
Bunch length & $100 \mathrm{fs}$ \\
Undulator parameter & $3.5-2.35$ \\
Photon energy & $282-533 \mathrm{eV}$
\end{tabular}

from the SwissFEL design value of $250 \mathrm{keV}$ to $460 \mathrm{keV}$. In the following discussion we assume the second method using the increased energy spread for the simulations.

The maximum tilt and therefore the shortest FEL pulse length is determined by the aperture in the undulator beamline in the tilt plane. Because the beam is aligned to the middle of either the first or second half of the electron bunch, the maximum offset is $75 \%$ of the transverse extension of the tilt. In the case of SwissFEL the aperture radius is $2.5 \mathrm{~mm}$ and thus the maximum tilt has a peak-to-peak amplitude of $3.3 \mathrm{~mm}$, with an extension of $0.8 \mathrm{~mm}$ to one side and $2.5 \mathrm{~mm}$ to the other. With some safety margin it can be assumed that a tilt of $2 \mathrm{~mm}$ is feasible, which is also consistent with the maximum deflection strength of the RF deflector upstream of the undulator beamline. Fig. 2 shows the beam size and the centroid position for a misaligned electron slice. The exemplary offset of $300 \mu \mathrm{m}$ in the figure corresponds to a tilt of $1.2 \mathrm{~mm}$ over the entire bunch: when one half of the bunch is aligned to the undulator axis the end points of that half have an offset of $\pm 300 \mu \mathrm{m}$. That offset must be sufficient to suppress the FEL amplification so that the beam quality in the second half of the bunch is preserved for the second stage. As shown in the next section, the selected tilt fulfills this condition. Note that at the turning point of the betatron oscillation the sawtooth oscillation of the centroid is larger than the beam size and the overlap between beam and radiation field is sufficiently disrupted. This is driven by the phase advance of

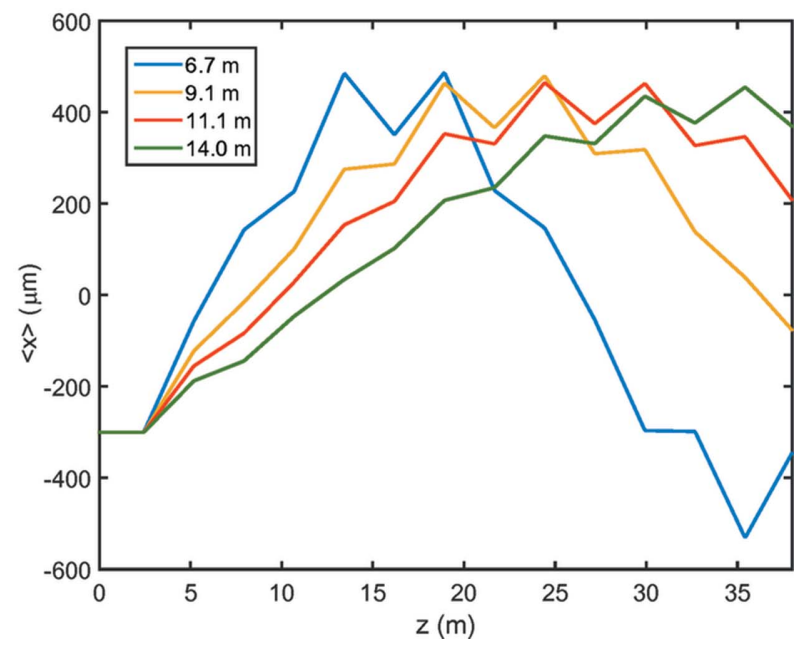

Figure 2

Beam size (left) and centroid position (right) of a misaligned electron slice for various focusing strengths, defined by their average betatron function value (see inserts). 
the betatron oscillation over one FEL gain length, which is the largest for the smallest average betatron-function value.

If needed the pulse length can be further reduced by placing an emittance spoiler foil with two slits in front of the undulator (Emma et al., 2010), where most of the tilted beam hits the foil outside of the slits and is scattered by the foil. In this way the emittance is increased, preventing the electrons from lasing except for those electrons which are passing the slits. However, this option is not necessary for the scheme proposed here and is thus not further discussed.

\section{FEL performance for tilted beam}

The key feature of the proposed method is the injection of a tilted beam into the undulator lattice. Depending on the strength of the focusing the electron beam slices undergo betatron oscillations, where they are misaligned with respect to the undulator axis. This oscillation separates the electron beam from the local radiation field and the associated effective loss in field amplitude prevents the beam slice from inducing microbunches and thus from coherent emission. Only the part where the tilted bunch crosses the undulator axis exhibits efficient FEL amplification because the degradation by the betatron oscillation does not occur there. Through the amplitude of the tilt the length of the FEL pulse can be controlled. Fig. 3 shows the pulse lengths for various tilts, operating at the $K$-edge of carbon at $282 \mathrm{eV}$. The simulation time-window is $50 \mathrm{fs}$ long, which is half of the envisaged bunch length to leave the same length available for the second pulse after the bunch has been delayed and realigned to the undulator axis. In the simulations with various tilts (shown in Fig. 3), two cases, i.e. with no tilt and with a tilt of $0.6 \mathrm{~mm}$, do not exhibit a pulse length which is confined to the $15 \mu \mathrm{m}$ beam section assigned for amplifying the first pulse. The residual FEL amplification, which occurs in the other section of the bunch, will spoil the beam quality there and thus affect the amplification in the second stage. For a clean two-pulse operation a tilt of at least $0.9 \mathrm{~mm}$ is needed at the given

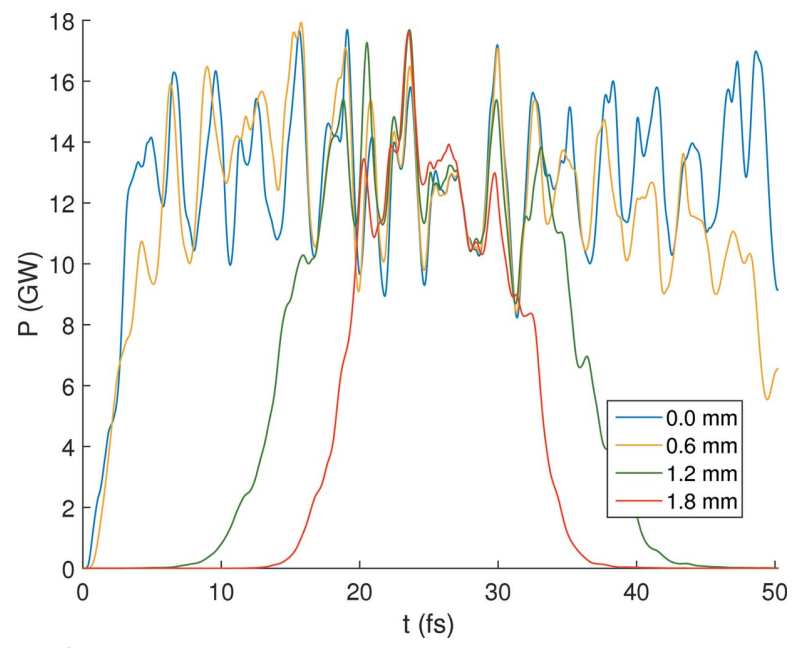

Figure 3

SASE-FEL radiation profile, averaged over 20 shots, at the end of the first undulator stage at a photon energy of $282 \mathrm{eV}$ for various beam tilts.
Table 2

The r.m.s. FEL pulse lengths for both stages for various beam tilts, based on 20 SASE-FEL runs each.

\begin{tabular}{lll}
\hline Tilt & Stage 1 & Stage 2 \\
\hline $0.0 \mathrm{~mm}$ & $13.91 \pm 0.35 \mathrm{fs}$ & $14.09 \pm 0.38 \mathrm{fs}$ \\
$0.6 \mathrm{~mm}$ & $13.18 \pm 0.34 \mathrm{fs}$ & $6.35 \pm 0.48 \mathrm{fs}$ \\
$1.2 \mathrm{~mm}$ & $7.03 \pm 0.30 \mathrm{fs}$ & $3.02 \pm 0.20 \mathrm{fs}$ \\
$1.8 \mathrm{~mm}$ & $4.42 \pm 0.28 \mathrm{fs}$ & $2.01 \pm 0.16 \mathrm{fs}$ \\
\hline
\end{tabular}

photon energy. The r.m.s. bunch lengths obtained for four different beam tilts, averaged over 20 independent SASE shots, are listed in Table 2.

Before the second stage the head part of the bunch has to be aligned to the undulator axis. Depending on the betatron phase advance in this first stage this requires a correction in offset and angle. The dipoles in the delaying chicane can perform this function in addition to their purpose of delaying the electron bunch in a controllable manner. Without any delay and with a $100 \mathrm{fs}$ bunch length the second pulse would be generated $50 \mathrm{fs}$ ahead of the first pulse. In the case of SwissFEL the chicane is designed to match the electron timeof-flight to that of the photon beam in the self-seeding configuration, which is about 1 ps. Therefore by tuning the magnet strength the arrival time of the second bunch can be controlled in the range between $50 \mathrm{fs}$ ahead of the first pulse and 950 fs after it. A given electron beam energy jitter would introduce a corresponding jitter in the delay. For the expected performance of SwissFEL this jitter is $0.01 \%$ (Ganter, 2012), which, even for the largest delay of $1 \mathrm{ps}$, is well below $1 \mathrm{fs}$.

The results for the second pulse, tuned to $533 \mathrm{eV}$, are shown in Fig. 4 and listed in Table 2. In comparison with the first pulse at $282 \mathrm{eV}$ the pulses are shorter and the energy is reduced. There are two reasons for this. First, to achieve a higher photon energy the undulator parameter is reduced from 3.5 to 2.35. This, in turn, increases the gain length and reduces the saturation power. Second, the optical mode size is smaller due to the reduced diffraction (Saldin et al., 1993). Consequently it requires less betatron motion to separate the electron beam

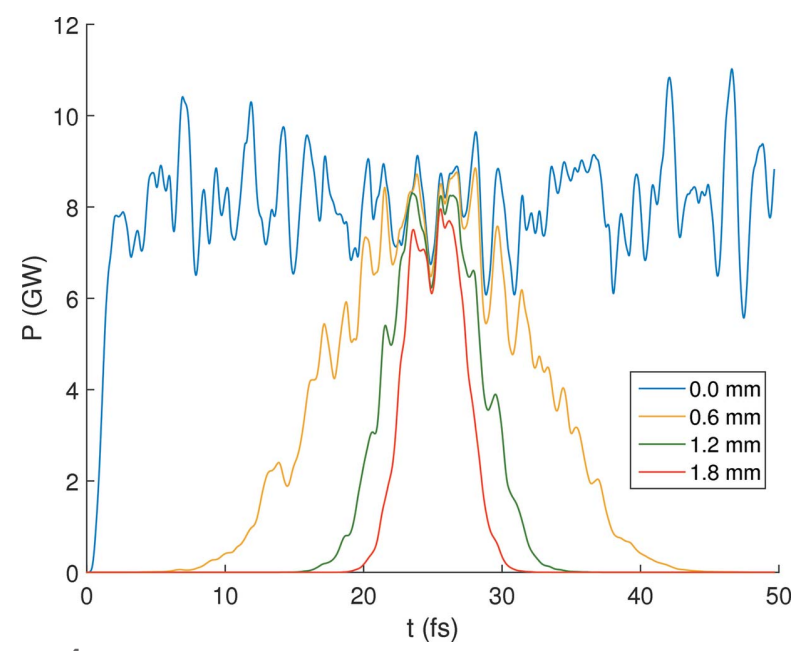

Figure 4

SASE radiation profile, averaged over 20 shots, at the end of the second undulator stage at a photon energy of $533 \mathrm{eV}$ for various beam tilts. 
Table 3

FEL pulse lengths for various focusing strengths.

\begin{tabular}{ll}
\hline Mean $\beta$-function & Pulse length \\
\hline $6.7 \mathrm{~m}$ & $3.02 \pm 0.20 \mathrm{fs}$ \\
$9.1 \mathrm{~m}$ & $4.66 \pm 0.35 \mathrm{fs}$ \\
$11.1 \mathrm{~m}$ & $6.86 \pm 0.50 \mathrm{fs}$ \\
$14.0 \mathrm{~m}$ & $10.51 \pm 0.56 \mathrm{fs}$ \\
\hline
\end{tabular}

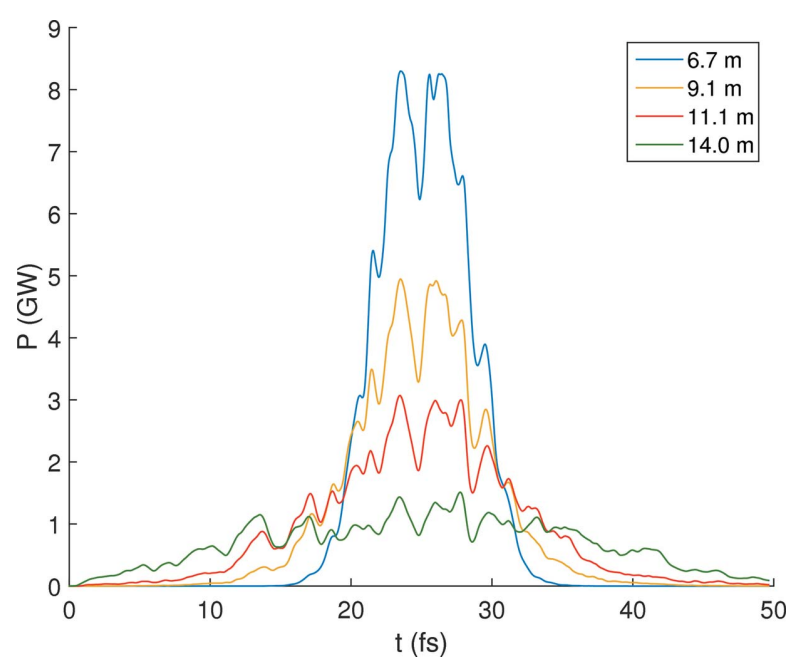

Figure 5

Average SASE pulse profile for various focusing strengths, as a function of the mean betatron function (in the legend) and a beam tilt of $1.2 \mathrm{~mm}$.

slice from the radiation field. However, it is possible to compensate the dependence of the pulse length on the photon energy for a given beam tilt with the mean betatron-function within the stage. In Fig. 5 and Table 3 the FEL pulse length for various focusing strengths are shown. Weaker focusing yields lower peak power but the pulse energy is nearly constant because lower power is compensated with longer bunches. With a rematching to a different average betatron-function value over the delaying chicane the pulse lengths of both pulses can be adjusted relative to each other.

\section{Conclusion}

We have presented a new method to generate two FEL pulses with independent control on the central photon energies and the relative delay between them. Our method uses a tilted electron beam and requires an undulator beamline with tunable gap and a delaying magnetic chicane. This scheme is not restricted to two colors alone but can be scaled up to $n$ colors with $n-1$ interleaved delaying chicanes similar to the two-color case. This comes at the price of increasing the undulator length accordingly to drive each pulse into saturation. The proposed scheme will be implemented in the soft X-ray beamline Athos at SwissFEL. It allows future users to choose two photon energies, which can differ by up to a factor of five, and a delay between -50 and $950 \mathrm{fs}$, to address their experimental needs. Some fine-tuning of the FEL pulse lengths and energy can be made with the degree of the beam tilt and the mean focusing strength in each stage of the undulator beamline. It opens up the possibility for new types of experiments which benefit from the high flexibility in setting up the two-color operation.

\section{Acknowledgements}

We are indebted to Thomas Schietinger, Terence Garvey, Rafael Abela and Hans Braun for helping to improve the content of the paper, both in language and content.

\section{References}

Allaria, E. et al. (2013). Nat. Commun. 4, 2476.

Campbell, L. T., McNeil, B. W. J. \& Reiche, S. (2014). New J. Phys. 16, 103019.

De Ninno, G., Mahieu, B., Allaria, E., Giannessi, L. \& Spampinati, S. (2013). Phys. Rev. Lett. 110, 064801.

Dlott, D. D. \& Fayer, M. D. (1989). J. Opt. Soc. Am. B, 6, 977-994.

Emma, P. et al. (2010). Nat. Photon. 4, 641-647.

Ganter, R. (2012). SwissFEL conceptual design report. Report 10-04 Paul Scherrer Institute, Switzerland.

Geloni, G., Kocharyan, V. \& Saldin, E. (2010a). Scheme for Femtosecond-Resolution Pump-Probe Experiments at XFELs with Two-Color Ten GW-Level X-ray Pulses. Report 10-004. DESY, Hamburg, Germany.

Geloni, G., Kocharyan, V. \& Saldin, E. (2010b). Scheme for Simultaneous Generation of Three-Color Ten GW-Level X-ray Pulses from Baseline XFEL Undulator \& Multi-User Distribution System for XFEL Laboratory. Report 10-006. DESY, Hamburg, Germany.

Geloni, G., Kocharyan, V. \& Saldin, E. (2011). Opt. Commun. 284, 3348-3356.

Hara, T., Inubushi, Y., Katayama, T., Sato, T., Tanaka, H., Tanaka, T., Togashi, T., Togawa, K., Tono, K., Yabashi, M. \& Ishikawa, T. (2013). Nat. Commun. 4, 2919.

Lutman, A. A., Coffee, R., Ding, Y., Huang, Z., Krzywinski, J., Maxwell, T., Messerschmidt, M. \& Nuhn, H. D. (2013). Phys. Rev. Lett. 110, 134801.

Lutman, A. A., Decker, F. J., Arthur, J., Chollet, M., Feng, Y., Hastings, J., Huang, Z., Lemke, H., Nuhn, H. D., Marinelli, A., Turner, J. L., Wakatsuki, S., Welch, J. \& Zhu, D. (2014). Phys. Rev. Lett. 113, 254801.

Mahieu, B., Allaria, E., Castronovo, D., Danailov, M. B., Demidovich, A., De Ninno, G., Di Mitri, S., Fawley, W. M., Ferrari, E., Fröhlich, L., Gauthier, D., Giannessi, L., Mahne, N., Penco, G., Raimondi, L., Spampinati, S., Spezzani, C., Svetina, C., Trovò, M. \& Zangrando, M. (2013). Opt. Express, 21, 22728-22741.

Marinelli, A., Lutman, A. A., Wu, J., Ding, Y., Krzywinski, J., Nuhn, H. D., Feng, Y., Coffee, R. N. \& Pellegrini, C. (2013). Phys. Rev. Lett. 111, 134801.

Marinelli, A., Ratner, D., Lutman, A. A., Turner, J., Welch, J., Decker, F. J., Loos, H., Behrens, C., Gilevich, S., Miahnahri, A. A., Vetter, S., Maxwell, T. J., Ding, Y., Coffee, R., Wakatsuki, S. \& Huang, Z. (2015). Nat. Commun. 6, 6369.

Petrillo, V. et al. (2013). Phys. Rev. Lett. 111, 114802.

Ratner, D. et al. (2015). Phys. Rev. Lett. 114, 054801.

Ravasio, A. et al. (2009). Phys. Rev. Lett. 103, 028104.

Reiche, S. (2000). Nucl. Instrum. Methods Phys. Res. A, 445, 139-142.

Ronsivalle, C. et al. (2014). New J. Phys. 16, 033018.

Saldin, E. L., Schneidmiller, E. A. \& Yurkov, M. V. (1993). Opt. Commun. 97, 272-290.

Schweigert, I. V. \& Mukamel, S. (2007). Phys. Rev. A, 76, 012504.

Shalaby, M. \& Hauri, C. P. (2015). Nat. Commun. 6, 5976.

Widmann, C., Afonso Rodriguez, V., Braun, N., Nicolai, M., Papash, A. R. M., Rossmanith, R., Saevert, A., Werner, W., Kaluza, M. C., Bernhard, A. \& Mueller, A.-S. (2014). Proceedings of the 2014 International Particle Accelerator Conference, pp. 2803-2806.

Yu, L. \& BenZvi, I. (1997). Nucl. Instrum. Methods Phys. Res. A, 393, 96-99. 